\title{
Nonlinearity in the dynamics of photoinduced nucleation process
}

\author{
Kunio Ishida \\ Corporate Research and Development Center, Toshiba Corporation \\ 1 Komukaitoshiba-cho, Saiwai-ku, Kawasaki 212-8582, Japan \\ and \\ Keiichiro Nasu \\ Solid State Theory Division, Institute of Materials Structure Science, KEK, \\ Graduate University for Advanced Study, and CREST JST, \\ 1-1 Oho, Tsukuba, Ibaraki 305-0801, Japan
}

\begin{abstract}
Nonlinear dynamics of photoinduced cooperative phenomena is studied by numerical calculations on a model of molecular crystals. We found that the photoinduced nucleation process is triggered only when certain amount of excitation energy is supplied in a narrow part of the system, i.e., there exists a smallest cluster of excited molecules which makes the nucleation possible. As a result, the portion of the cooperatively converted molecules is nonlinearly dependent on the photoexcitation strength, which has been observed in various materials.
\end{abstract}


The discovery of the photoinduced cooperative phenomena has attracted our attention to new aspects of the nonequilibrium dynamics of excited states in condensed matter[1]. They have mentioned that the dynamics of photoinduced domain growth is a key to understand the physics of the photoinduced cooperativity, and many experimental and theoretical studies have been performed to reveal the mechanism of these phenomena $[2,3$, 5, 6, 7, 4, 8, 9, [10, 11. Above all, interdomain interaction and/or nonlinearity in the pattern formation process of photoinduced domains is important to understand their dynamical properties.

As for the theoretical study of these phenomena, we have proposed a model of molecular crystals to describe the initial nucleation processes of photoinduced domains [10, 11]. We found that an initially excited molecule becomes a nucleus of a photoinduced domain, and that its growth dynamics is understood by the numerical solutions of the time-dependent Schrödinger equation. Although we discussed the interdomain interactions in some special cases[10], no microscopic theories on the nonlinearity of the domain growth dynamics have been obtained so far.

Nonlinear dynamics of spatio-temporal pattern formation has been extensively studied to understand the various aspects of nonequilibrium phenomena in wide scale-range, e.g., from nanoscale of the Belousov-Zhabotinsky reaction or the phase separation dynamics in the kinetic Ising model[12] to the astronomical scale of cosmic structure formation[13]. It has been pointed out that the density fluctuation of relevant physical properties is a "seed" of growing patterns, and that the initial density distribution determines their complicated structure. Hence, in analogy to them, it is required to study the domain growth dynamics in the presence of excitation density fluctuation in order to clarify and understand the nonlinear nature of the photoinduced cooperativity.

In this Letter, we study the photoinduced nucleation processes focusing on the relation between the excited energy distribution and the pattern formation dynamics. We employ a model of molecules arrayed on a square lattice[10, 11, and show that the spatial fluctuation of excitation density plays a key role in the growth/extinction processes of photoinduced domains as in the case of various types of phase separation dynamics[14].

To describe the present model, we first point out that one of the elementary processes of the photoinduced nucleation in this system is the nonadiabatic transition between the ground and the excited electronic states in each molecule. Electrons relevant to this process are assumed to be localized in each molecule, and two electronic levels corresponding to the ground and excited electronic states are taken into account per molecule. The diabatic potential energy surfaces with a single relevant vibration mode are assumed in each molecule which cross with each other and the nonadiabaticity in the dynamics is taken into account via "spin-flip" interaction between two electronic states as in the studies of the nonadiabatic transitions in typical organic molecules [15]. As for the intermolecular interaction, we take into account the bilinear coupling terms between distortion of adjacent molecules and the dipole-dipole interaction between ex- 
cited state electrons of which the strength varies with the molecular distortion. Furthermore, the interaction which describes the molecular distortion induced by the excited state electrons in the adjacent molecules is also considered. Hence, the Hamiltonian in the present study is described by:

$$
\begin{aligned}
\mathcal{H} & =\sum_{\vec{r}}\left\{\frac{p_{\vec{r}}^{2}}{2}+\frac{\omega^{2} u_{\vec{r}}^{2}}{2}+\left(\sqrt{2 \hbar \omega^{3}} s q_{\vec{r}}+\varepsilon \hbar \omega+s^{2} \hbar \omega\right) \hat{n}_{\vec{r}}+\lambda \sigma_{x}^{\vec{r}}\right\} \\
& +\sum_{\left\langle\vec{r}, \overrightarrow{r^{\prime}}\right\rangle}\left[\alpha \omega^{2}\left(u_{\vec{r}}-\beta \hat{n}_{\vec{r}}\right)\left(u_{\overrightarrow{r^{\prime}}}-\beta \hat{n}_{\overrightarrow{r^{\prime}}}\right)-\left\{V-W\left(u_{\vec{r}}+u_{\overrightarrow{r^{\prime}}}\right)\right\} \hat{n}_{\vec{r}} \hat{n}_{\overrightarrow{r^{\prime}}}\right],
\end{aligned}
$$

where $p_{\vec{r}}$ and $u_{\vec{r}}$ are the momentum and coordinate operators for the vibration mode of a molecule at site $\vec{r}$, respectively. The electronic states at site $\vec{r}$ are denoted by $|\downarrow\rangle_{\vec{r}}$ (ground state) and $|\uparrow\rangle_{\vec{r}}$ (excited state) and $\sigma_{i}^{\vec{r}}(i=x, y, z)$ are the Pauli matrices which act only on the electronic states of the molecule at site $\vec{r} . \hat{n}_{\vec{r}}$ denotes the density of the electron in $|\uparrow\rangle_{\vec{r}}$ which is rewritten as $\hat{n}_{\vec{r}}=\sigma_{z}^{\vec{r}}+1 / 2$. The second sum which gives the intermolecular interaction is taken over all the pairs on nearest neighbor sites, and the vibrational period of an individual molecule is denoted by $T=2 \pi / \omega$. The vibration modes are quantized in order to describe the nonadiabatic transition between potential energy surfaces rigorously.

We chose the values of the parameters as: $\varepsilon=2.3, s=1.4, V=1.1, W=0.2, \alpha=$ $0.1, \beta=0.2$, and $\lambda=0.2$. Although those values are typical for organic molecules as for electron-vibration coupling [16] and the intermolecular Coulomb interaction, we mention that the other parameters are not easy to determine their values either from theoretical calculations or experimental results.

The numerical solutions of the time-dependent Schrödinger equation for the Hamiltonian (11) were obtained by the Runge-Kutta method with various initial conditions. In each series of simulations, the dynamics of the system on a $96 \times 96$ lattice with periodic boundary conditions was calculated. We also applied a mean-field approximation in which the contribution of the wavefunction at the nearest neighbor sites is substituted by the average value with respect to the wavefunction $|\Phi(t)\rangle$, which is also quite suitable for large scale computing with a grid environment, for example. The details of the model and the calculation method are described in Ref. [11].

We have pointed out that the population of the excited electronic state $\tilde{N}(\vec{r}, t)=\left\langle\Phi(t)\left|\hat{n}_{\vec{r}}\right| \Phi(t)\right\rangle$ is suitable to discuss the dynamics of the spatial patterns of the photoinduced domains. In particular, the sum of the excited state population $N(t)=\sum_{\vec{r}} \tilde{N}(\vec{r}, t)$ gives a measure to estimate the size of the photoinduced domain[11], which is shown in Fig. 1 as functions of time for several initial conditions. The dotted line in Fig. 1 shows that an isolated excited molecule does not trigger the nucleation processes but loses the excitation energy after a few periods of the molecular vibration mode. When two excited molecules are placed on the nearest neighbor 
sites, the potential energy barrier between $|\downarrow\rangle_{\vec{r}}$ and $|\uparrow\rangle_{\vec{r}}$ in these molecules are still too high to make the electronic state conversion possible. Thus no domain growth occurs though these molecules do not go back to the ground state within the simulation time(see the dot-dashed line in Fig. 1). On the contrary, when the initially excited molecules form an $I$-tromino or an $I$-tetromino, $N(t)$ increases as the excitation energy is transferred to the other molecules, and a photoinduced domain grows as shown in the solid line and the dashed line in Fig. 1 .

The above results clearly show that there exists a smallest cluster of excited molecules which leads to the domain growth after photoexcitation, i.e., a certain amount of excitation energy is required to be initially concentrated in a narrow spatial area of the system. The dynamics of the photoexcited states differs qualitatively from each other as the initial state is varied, and, in particular, the size of the domain is not simply determined by the value of $N(0)$. To be more precise, the domain growth dynamics reflects the symmetry of the initial configuration as well as the concentration of excitation energy. A taxonomic study of the domain growth dynamics with respect to the configuration of initial excited molecules will be given elsewhere.

Although the size of the smallest cluster for the domain growth depends on the values of the parameters, we also stress that our simple model which consists only of localized electrons and molecular vibration modes is sufficient to discuss the nonlinearity of the nucleation process.

We extended our calculations to the cases where initially excited molecules are distributed at random, and studied the pattern formation dynamics of excited-state domains during the initial nucleation processes in coherent regime. In this case, the excitation ratio $\rho$, defined by the ratio of the number of the initially excited molecules to the total number of the molecules, is a relevant parameter. Figure 2 shows the gradation maps of $\tilde{N}(\vec{r}, t)$ for $t=0, t=7.5 T$, and $t=15 T$ for $\rho=0.0625$. At $t=0$ the distribution of the excited molecules is not uniform, and thus the fluctuation of $N(\vec{r}, t)$ is present. When excited molecules are densely concentrated in certain part of the system, the molecules around them are able to overcome the potential energy barrier to make the electronic state conversion, and thus the excited-state domain starts to grow there. On the contrary, when the density of the excited molecules is not sufficiently high for the domain growth, the excitation energy is released to the other molecules through the vibrational coupling $\alpha$, and thus the excited molecules return to the ground state. As a result, we obtain islands of photoinduced domains shown in Fig. 2-(b) around the parts of the system which are initially supplied with higher excitation energy. These islands merge with each other to make larger ones as shown in Fig. 2-(c), and $N(t)$ continues to increase with time. Thus, the density fluctuation of the initially excited molecules reflects the structure of the photoinduced domains, and vice versa. We note that these results directly reflects the discussion on the smallest cluster for the domain growth shown previously.

We also calculated the typical correlation length $R$ for the photoinduced domains. To obtain 
$R$ we calculated the two-point correlation function

$$
C(|\vec{r}|, t)=\sum_{\overrightarrow{r^{\prime}}}\left(\left\langle\hat{n}_{\vec{r}+\overrightarrow{r^{\prime}}} \hat{n}_{\overrightarrow{r^{\prime}}}\right\rangle-\left\langle\hat{n}_{\vec{r}+\overrightarrow{r^{\prime}}}\right\rangle\left\langle\hat{n}_{\overrightarrow{r^{\prime}}}\right\rangle\right)
$$

where $\langle.$.$\rangle denotes the average over 64$ series of simulation for a fixed value of $\rho$. In each series of simulation, the initial excited molecules are in different configurations. Then we define $R$ by the relation $C(R, t)=C(0, t) / e$, and $R$ in units of the lattice constant is shown in Fig. 3 for $\rho=0.03,0.05$, and 0.1 . All of the three lines in Fig. 3 show that $R$ starts to increase in the same manner, since $R$ for $t \sim 0$ is determined by the individual clusters of excited molecules. Although Fig. 3 shows that $R$ is proportional to $t$ for small values of $r$ as in the case of a single domain[11], $R$ increases more slowly for $\rho=0.1$. This difference is due to the interference between growing domains, i.e., a domain disturbs the growth of the other ones after they share perimeters with each other. Hence, the increase rate of $R$ slows down and it seems to behave as $t^{\alpha}$ for $\alpha<1$ as in the diffusive domain growth[12], for example. However, we stress that the dynamics considered in the present study is always in coherent regime, and thus the slowdown of the growth rate is, as it were, "false" $t$-dependence of $R$. Such a behavior of $R$ should be discriminated from the diffusive domain growth in the systems which belong to a different universality class. In particular, since these properties reflects on the structure factor which is the Fourier transformation of $C(|\vec{r}|, t)$, they can be distinguished from each other experimentally by varying the excitation ratio.

We also found that the size of the photoinduced domains nonlinearly depends on the excitation ratio $\rho$. Figure 4 shows the conversion rate $c_{\rho}$ defined by $c_{\rho}=N(t=15 T) / M$ as a function of $\rho$, where $M$ is the number of molecules and is $9216\left(=96^{2}\right)$ in the present calculations. To obtain these results we calculated the average value of $c_{\rho}$ for each value of $\rho$ over 64 series of simulations as in the calculation of $R$.

Figure 4 shows that $c_{\rho}$ depends on $\rho$ as $\sim \rho^{3}$ in the dilute limit $(\rho \sim 0)$, and deviates from $\rho^{3}$ for $\rho>0.1$. This feature reflects the size of the smallest cluster which enables domain growth. With a fixed value of $\rho$, the smallest clusters for domain growth (a tromino) appear in the initial state with a probability proportional to $\rho^{3}$ in the dilute limit. Hence, only a portion of the initially excited molecules contributes to the domain growth. As we can neglect the interference between domains for $\rho \sim 0$, the number of converted molecules is proportional to $\rho^{3}$ in this case.

As $\rho$ increases, the growing domains interfere with each other and the growth rate becomes lower as shown in Fig. 3. Thus $c_{\rho}$ deviates from $\rho^{3}$ as the value of $\rho$ increases as shown in Fig. 4 . In any case, the conversion rate increases as $\rho^{m}$ where $m$ is the size of the smallest cluster which triggers the nucleation processes. If $m$ is experimentally determined through the measurement of optical properties, for example, we will have a clue to understand the microscopic mechanism of the elementary process of the domain growth dynamics. 
Figure 4 also shows that $c_{\rho} / \rho<7$ in the present case, although some larger values were reported in experimental studies [2, 3, 4, 5]. Larger values of $c_{\rho} / \rho$ were found in materials close to their critical temperature, since instability of thermodynamical state of the system enhances the conversion rate. Hence, we mention that the present results correspond to the cases away from the critical temperature. Furthermore, we should note that the present calculations are valid before the decoherence of vibrational states takes place, and that the value of $c_{\rho}$ shown in Fig. 4 corresponds to that for $t \sim 3$ psec for $T \sim 200$ fsec as in typical organic molecules. We, however, expect that the domains continue to grow after the decoherence occurs, and thus the experimentally obtained conversion rate cannot be directly compared with the present results quantitatively. We stress that the nonlinearity of conversion rate as a function of $\rho$ is essentially understood by the initial process of the domain growth, and that the present calculations are of importance in order to understand the microscopic mechanism of the photoinduced cooperativity.

Summarizing the present Letter, we conclude that the nonlinear dynamics of the photoinduced domain growth is understood by a model of localized electrons coupled with molecular vibration mode shown by Eq. (11). We found that there exists a smallest cluster of excited molecules which is required to induce the domain growth, and that the initial nucleation process does not start unless sufficient energy is concentrated in a narrow area of the system. When the fluctuation of excitation density realizes the formation of such clusters, photoinduced nucleation is triggered to form domains afterwards, and thus the excitation energy fluctuation determines the domain structure. We also showed that the nonlinearity of the conversion rate as a function of excitation ratio is understood as a result of the interdomain interactions besides the above effect.

K. I. is grateful to K. Takaoka, H. Asai, and S. Nunoue for helpful advice. This work was supported by the Next Generation Super Computing Project, Nanoscience Program, MEXT, Japan, and the numerical calculations were carried out on the computers at the Research Center for Computational Science, National Institutes of Natural Sciences.

\section{References}

[1] Photoinduced phase transitions, edited by K. Nasu (World Scientific, Singapore, 2004).

[2] S. Koshihara et al., J. Phys. Chem. B 103, 2592 (1999).

[3] S. Koshihara et al., Phys. Rev. B 52, 6265 (1995).

[4] S. Iwai et al., Phys. Rev. Lett. 88, 057402 (2002).

[5] A. Mino et al., Mol. Cryst. Liq. Cryst. 314, 107 (1998). 
[6] N. O. Moussa et al., Phys. Rev., Lett., 94, 107205 (2005).

[7] J. S. Costa et al., J. Phys. Conf. Ser. 21, 67 (2005).

[8] K. Koshino and T. Ogawa, Phys. Rev. B58, 14804 (1998).

[9] H. Mizouchi and K. Nasu, J. Phys. Soc. Jpn. 69, 1543 (2000).

[10] K. Ishida, Phys. Stat. Sol. c3, 3438 (2006).

[11] K. Ishida and K. Nasu, Phys. Rev. B76, 014302 (2007).

[12] S. van Gammert, et al., Phys. Rev. E72, 046131 (2005).

[13] V. Springel, et al., Nature 435, 629 (2005).

[14] P. C. Hohenberg and B. I. Halperin, Rev. Mod. Phys. 49, 435 (1977).

[15] L. Salem, Science 191, 822 (1976).

[16] K. Horikoshi et al., Opt. Commun. 259, 723 (2006). 


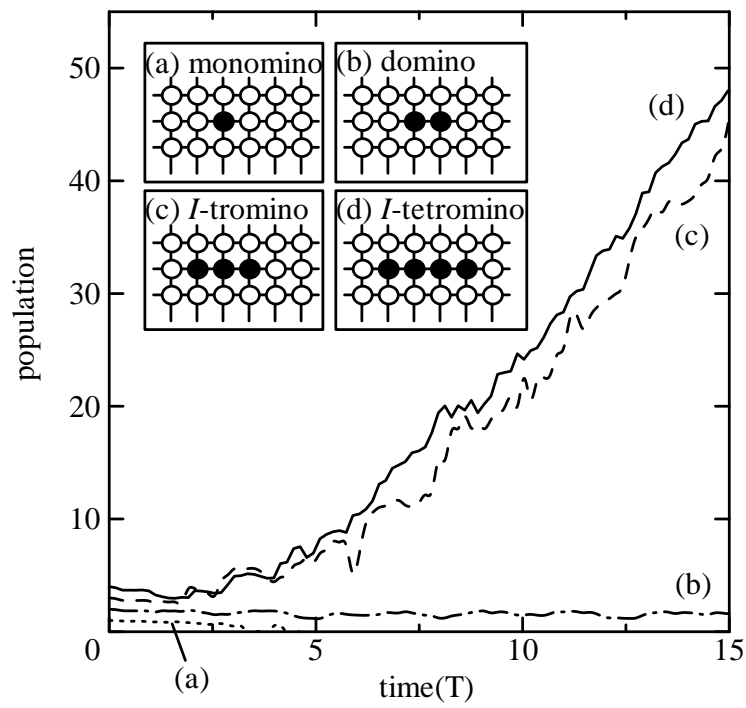

Figure 1: Population of the excited state molecule $N(t)$ as functions of time. Inset: initial configurations of excited molecules. the filled circles and the open circles correspond to the molecules in the Franck-Condon state and the ground state, respectively. 


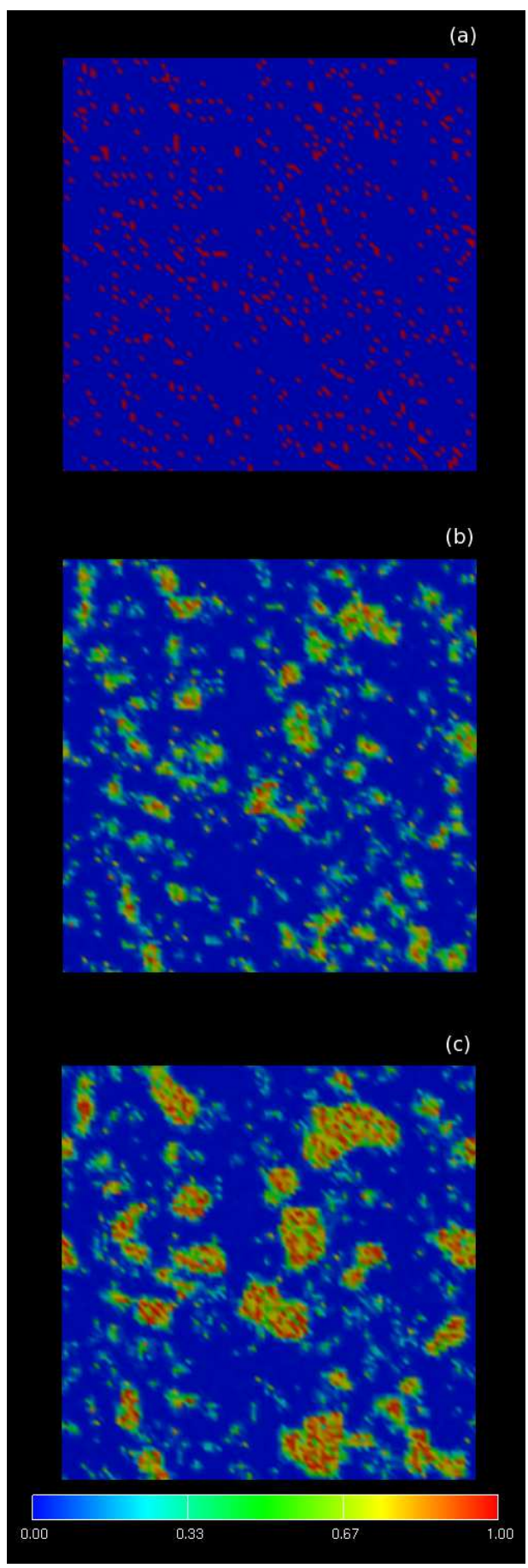

Figure 2: Gradation maps of excited state population $\tilde{N}(\vec{r}, t)$ on a $96 \times 96$ lattice for $\rho=0.0625$ for (a) $t=0$, (b) $t=7.5 T$, and (c) $t=15 T$. 


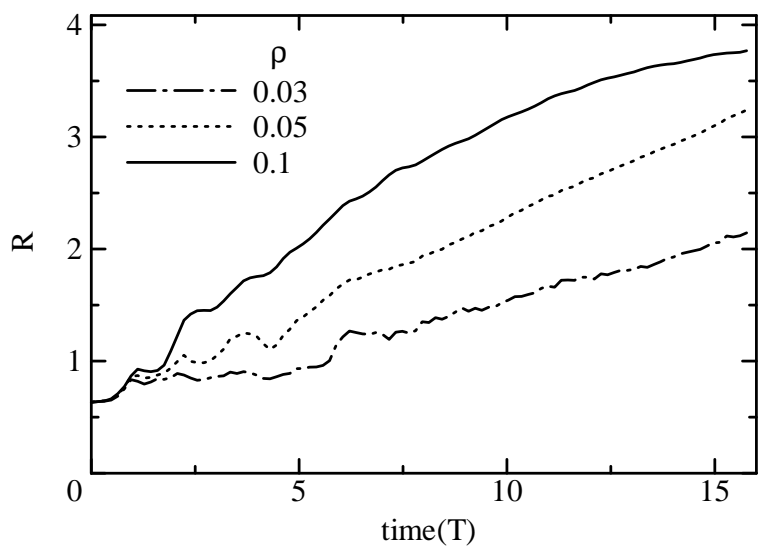

Figure 3: Correlation length $R$ in units of the lattice constant for $\rho=0.03,0.05$, and 0.1 as functions of time.

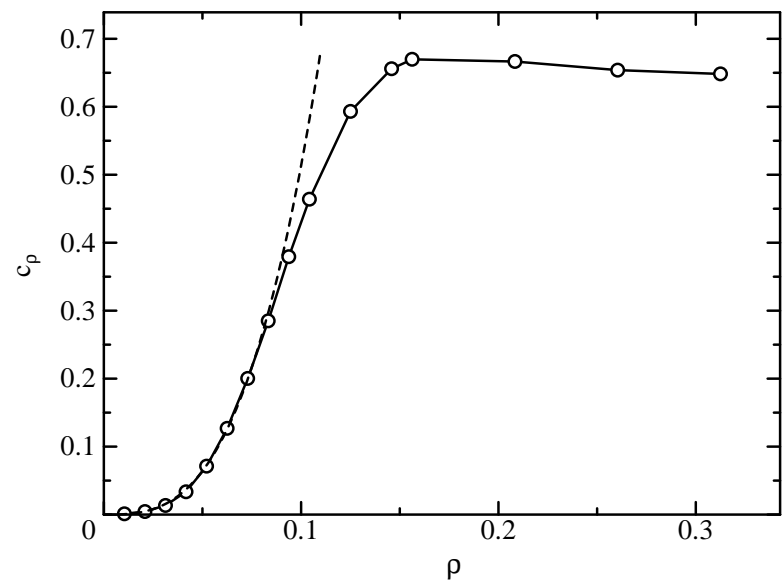

Figure 4: Conversion rate $c_{\rho}$ as a function of excitation ratio $\rho$. The dashed line which is proportional to $\rho^{3}$ is drawn as a guide for the eyes. 